\title{
ESTUDIO DE LA VIABILIDAD TÉCNICA DE UN SISTEMA DE CAPTACIÓN DE ENERGÍA PIEZOELÉCTRICA CON APLICACIÓN EN PLANTILLAS DE CALZADO DEPORTIVO
}

\author{
Fiama Vanessa norabuena Aliaga* \\ https://orcid.org/0000-0002-6619-419X \\ Universidad de Lima
}

Recibido: 16 de agosto del 2020 / Aprobado: 21 de octubre del 2020

doi: https://doi.org/10.26439/ing.ind2021.n40.5151

RESUMEN: La presente investigación propone el desarrollo de un sistema de captación de energía generada a partir de pisadas humanas con aplicación en plantillas de calzado deportivo. Se utilizaron los conceptos de energy harvesting y el fenómeno de la piezoelectricidad para la fabricación del prototipo compuesto por un sistema de once sensores piezoeléctricos y una plantilla impresa en 3D con el filamento flexible PLA. El prototipo se puso a prueba al simular las pisadas logrando generar hasta $1 \mathrm{~V}$ al encender un diodo LED. Sin embargo, la energía generada no fue la suficiente para cargar una unidad de almacenamiento de baja potencia.

PALABRAS CLAVE: fuentes de energías renovables no convencionales / sistemas de captación de energía / piezoelectricidad / calzado deportivo

\section{A TECHNICAL FEASIBILITY STUDY OF A PIEZOELECTRIC ENERGY HARVESTING SYSTEM APPLIED TO SPORTS SHOE INSOLES}

ABSTRACT: This research proposes the development of an energy harvesting system applied to sports shoe insoles, which uses human footsteps for energy generation. The concepts of energy harvesting and the phenomenon of piezoelectricity were used to manufacture a prototype consisting of a system with eleven piezoelectric sensors and a 3D printed insole made from PLA material. The prototype was tested using simulated footsteps, thus generating up to $1 \mathrm{~V}$ when an LED diode turned on. However, the energy generated was not enough to charge a low-power storage unit.

KEYWORDS: non-conventional renewable energy sources / energy harvesting system / piezoelectricity / sports shoe

*Correo electrónico: fiamanorabuena@gmail.com 


\section{INTRODUCCIÓN}

La creciente demanda energética y el fuerte impacto sobre el medio ambiente han originado que diversas investigaciones propongan nuevas formas de generación de energía que combinen la acción de caminar y una fuente a escala pequeña como la piezoelectricidad. A este concepto se le conoce como energy harvesting el cual se define como el proceso de recolección de energía residual que se origina naturalmente al presionar sensores piezoeléctricos ubicados en una plantilla, para luego ser convertida en energía eléctrica, almacenada en una unidad y utilizada como fuente de energía en diversas aplicaciones.

Ahmad y Hashim (2011) hicieron una plantilla captadora de energía con un parche piezoeléctrico para producir energía a partir de vibración constante al caminar y correr, cargando así una batería de 40 mAh a 1,2 V en 3750 segundos. Xie y Cai (2015) construyeron un mecanismo deslizante trapezoidal, en la suela de una zapatilla, que reportó, aproximadamente, $1 \mathrm{~W}$ de generación de energía. Fan et al. (2017) diseñaron un recolector piezoeléctrico que aprovecha el golpe del pie en el suelo al caminar, el balanceo de la pierna durante el caminar y la compresión del peso de la persona, generando 0,03 MW a 0,35 MW al caminar en velocidades de 2 a 8 km/h, respectivamente. Dewangan y Dubey (2017) elaboraron un sistema con 12 sensores piezoeléctricos ubicados en una plantilla, cuyo voltaje resultante fue amplificado para generar $6 \mathrm{~V}$ al caminar. Jiménez Martínez, de Frutos, Alonso y Vázquez (2015) optimizaron la captación de energía piezoeléctrica en un entorno de vibración, logrando la carga de un condensador de 200 pF en 3824 segundos.

\section{MATERIALES Y MÉTODOS}

Se recuperó la energía siguiendo cuatro fases fundamentales. También se consideró el concepto de ingeniería inversa como parte de la fase 4 de aplicación del proyecto.

\subsection{Fase 1. Sistema de captación de energía}

Para la presente investigación se eligió el sensor piezoeléctrico PZT de diafragma cerámico. El diseño propuesto, que se observa en la figura 1, consiste en once discos piezoeléctricos PZT soldados en paralelo.

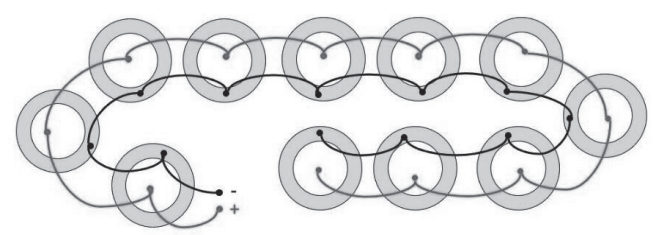

Figura 1. Sistema piezoeléctrico conectado en paralelo (pie izquierdo)

Elaboración propia 


\subsection{Fase 2. Acondicionamiento}

\subsubsection{Encendido del diodo LED}

Se hizo una prueba rápida de funcionamiento del soldado de los sensores piezoeléctricos, al prender un diodo LED, para indicar que el circuito estaba activo, probando así que el circuito conectado sí generaba energía.

\subsubsection{Carga de una unidad de almacenamiento}

La energía se almacena en tres etapas que son de entrada, proceso o control y salida. Esta puede cambiar dependiendo de la unidad a almacenar. La etapa de entrada está compuesta por el sistema de sensores piezoeléctricos encargados de captar la energía generada al caminar.

Dicha energía es de carácter alterno por lo que en la etapa de proceso o control se rectifica la señal con un puente de diodos. Seguido de un condensador que tiene la función de actuar como filtro para que el voltaje directo producido se mantenga constante (Maiwa, 2016) y se evite las variaciones de tensión (Jiménez Martínez et al., 2015).

Finalmente, en la etapa de salida, se carga una batería. Los detalles del almacenamiento de energía de la etapa de salida se observan en la fase 3.

\subsection{Fase 3. Almacenamiento}

Según la potencia, la energía que se requiera y el sistema de cosecha de energía, se pueden cargar tres tipos de unidad de almacenamiento como los condensadores, supercondensadores y las baterías.

El primer tipo es el condensador o capacitor, dispositivo que almacena energía y que consiste en dos placas de material conductor ubicado entre un aislador (Fluke Corporation, 2020). El segundo, el supercondensador, también llamado ultracapacitador, tiene un funcionamiento muy similar al de un condensador. La diferencia radica en que posee una mayor capacidad de almacenamiento a comparación de los condensadores comunes (medida en faradios) (Twenergy, 2019). Finalmente, la batería es un dispositivo que convierte la energía química contenida en sus materiales activos directamente en energía eléctrica por medio de una reacción electroquímica de oxidación-reducción (Mezei, 2011).

\subsection{Fase 4. Aplicación}

La aplicación del sistema de captación de energía piezoeléctrica es una plantilla, cuyo prototipo se fabricó con la tecnología de diseño, impresión y material 3D. La plantilla y el sistema piezoeléctrico se pusieron a prueba al encender un diodo LED de color rojo. 


\subsubsection{Diseño de la plantilla}

El prototipo se hizo al escanear las medias de un pie talla 38, para su posterior reconstrucción 3D y elaboración de la plantilla. El proceso de diseño consta de tres fases: adquisición de la información geométrica, reconstrucción de superficies y construcción de la plantilla.

\subsubsection{Fase 1. Adquisición de la información geométrica}

La información geométrica del pie de talla 38 se obtuvo al escanear el pie y generar una malla poligonal en el software empleado. Se necesitó el escáner 3D HandyScan 700 y el software VXelements. El resultado fue una superficie con la información geométrica del pie, canilla y pantorilla.

\subsubsection{Fase 2. Reconstrucción de superficies}

Se utilizó el software VXmodel para la reconstrucción de la forma del pie, a partir de la información geométrica escaneada, para así poder generar la malla poligonal.

Una vez obtenida la malla poligonal, esta fue filtrada, es decir, que se limaron manualmente superficies ajenas y puntos defectuosos que se observan en los dedos del pie. Posteriormente, se usó el software Geomagic Freeform para realizar mejoras más específicas de la malla poligonal al crear un cuerpo sólido que permitiera el moldeado en arcilla, lo cual hizo más fácil el diseño de la plantilla. Los dedos del cuerpo sólido se rellenaron con la función "Esculpir" para obtener una superficie uniforme y evitar las curvaturas innecesarias de los dedos del pie. El resultado se puede observar en la figura 2.

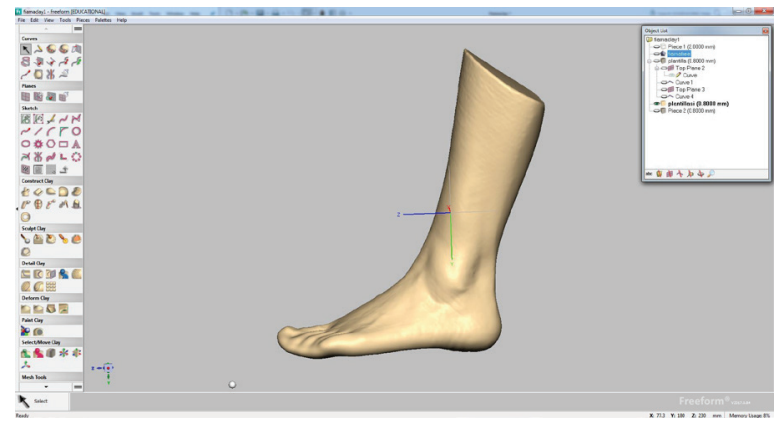

Figura 2. Rellenado de los dedos del pie

Elaboración propia 


\subsubsection{Fase 3. Construcción de la plantilla}

El cuerpo sólido, tratado anteriormente, se utilizó como molde para la extrusión de la base de la plantilla y formar dos superficies independientes. La extrusión fue de 3 a $5 \mathrm{~mm}$. Se utilizó el comando "Curve" y se mantuvo presionado con el comando "Fitting" para dibujar el contorno de la plantilla en la superficie de la planta del pie.

Las superficies fueron separadas por el comando "Boolean remove" para trabajar únicamente con la base de la cual saldrá la plantilla. El comando elegido remueve una figura de otra.

Las curvas que se presentan en el pie fueron proyectadas a una superficie plana con ayuda de la herramienta "Proyectar superficie a plano", obteniendo como resultado una plantilla con la forma actual del pie escaneado que se observa en la figura 3.

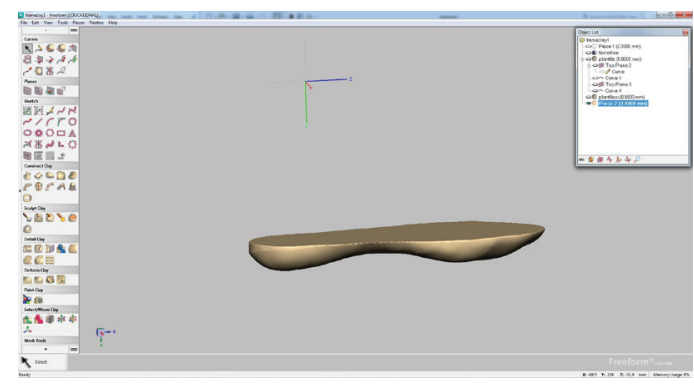

Figura 3. Proyección de la planta del pie

\section{Elaboración propia}

Se dibujaron con la opción de "Sketch", 11 círculos de 27,5 mm de diámetro y 1,5 mm de espesor para la ubicación de los sensores piezoeléctricos. También se hicieron dos huecos con la opción "Extruir" para poder conectar los cables con el protoboard. Por último, con la opción "Pipe" se hicieron surcos de $3 \mathrm{~mm}$ de ancho para colocar los cables y mantenerlos ordenados. El diseño final se observa a continuación en la figura 4.

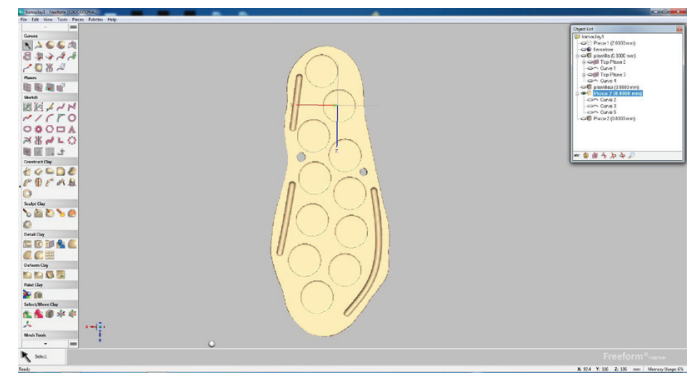

Figura 4. Dibujo círculos, huecos y surcos

Elaboración propia 


\subsubsection{Prototipo y sistema de captación de energía}

Como se puede observar en la figura 5 , el sistema de once sensores piezoeléctricos en paralelo fue montado encima de la plantilla y fue pisado simulando la forma de caminar para así poder encender el diodo LED y demostrar que el sistema piezoeléctrico sí genera energía.

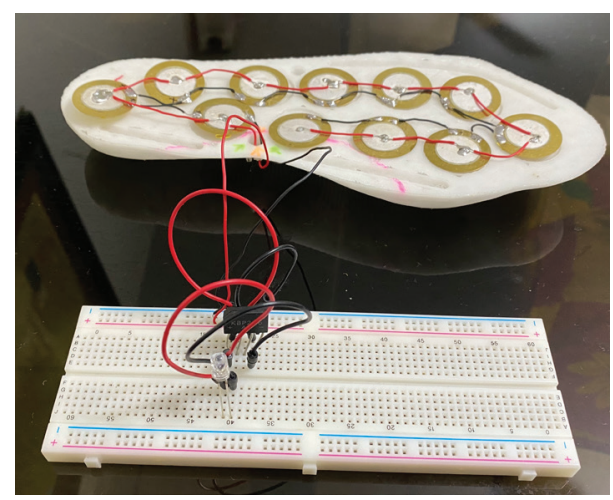

Figura 5. Prototipo para la prueba de encendido del diodo LED

Elaboración propia

\section{RESULTADOS}

\subsection{Diseño de la plantilla e impresión 3D}

En la figura 6, se observa la impresión acabada del prototipo cuya textura es dura y suave, sin embargo, no es flexible al doblar. Los sensores piezoeléctricos no pudieron ser insertados en los espacios previstos debido a la rigidez de la estructura. También, se observan surcos en los bordes de la plantilla, en los que se esperaba poner los cables para tener un mejor orden, sin embargo, no se pudieron utilizar, ya que las formas de los cables no pudieron ser ubicadas dentro del surco, pues las conexiones podían verse afectadas.

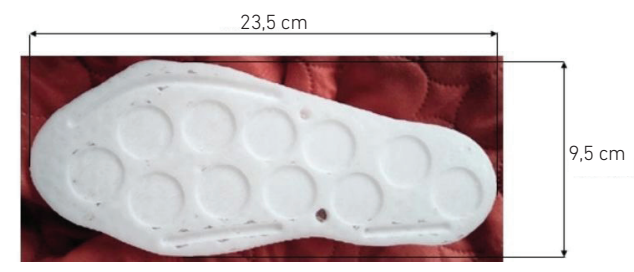

Figura 6. Impresión 3D del prototipo de la plantilla

Elaboración propia 


\subsection{Prototipo y sistema de captación de energía}

\subsubsection{Encendido del diodo LED}

La prueba se llevó a cabo en la oscuridad para lograr distinguir el color rojo del diodo que se prende, por unos segundos, justo cuando la persona va a levantar el talón para dar el siguiente paso. El diodo LED de color rojo trabaja con una corriente de $20 \mathrm{~mA}$ y un voltaje de 1,8 V (Vishay Semiconductors, 2020). Con un multímetro digital, se midió el voltaje generado por el sistema de once piezoeléctricos que fue de $1 \mathrm{~V}$, aproximadamente.

\subsubsection{Carga de una unidad de almacenamiento}

El arreglo, bastante simple y económico del sistema piezoeléctrico, no fue suficiente para la carga de un capacitor, cuyas especificaciones requerirían de $10 \mu \mathrm{F}$ a $16 \mathrm{~V}$ para la carga de este. Ahmad y Hashim (2011) detallan que la mayoría de los investigadores se están centrando en la aplicación de golpe de talón con piezoeléctrico. Sin embargo, la corriente eléctrica y/o los voltajes generados son muy bajos.

Al no generarse energía suficiente, se optó por tomar como referencia los resultados de artículos científicos y tesis de repositorio. En la tabla 1, se observan ejemplos de la literatura revisada y sus propuestas de pruebas experimentales, según la unidad de almacenamiento de energía.

Tabla 1

Resumen de los tipos de pruebas y resultados experimentales según trabajo de investigación

\begin{tabular}{|c|c|c|c|}
\hline Tipo de prueba & Artículo científico o tesis & Autor(es) & Resultados \\
\hline $\begin{array}{l}\text { Simulación del movi- } \\
\text { miento humano al co- } \\
\text { rrer en una trotadora a } \\
\text { la velocidad de } 5 \mathrm{~km} / \mathrm{h}\end{array}$ & $\begin{array}{l}\text { Desarrollo de un dispositivo } \\
\text { colector de energía que utiliza } \\
\text { materiales piezoeléctricos }\end{array}$ & $\begin{array}{l}\text { Robiah Ahmad y } \\
\text { Mohd Hashim }\end{array}$ & $\begin{array}{l}\text { Carga de batería NiMH } \\
40 \text { mAh a } 1,2 \text { V en } \\
3750 \text { segundos }\end{array}$ \\
\hline $\begin{array}{l}\text { Pruebas de medición } \\
\text { de fuerza con pesas de } \\
\text { precisión del piezocerá- } \\
\text { mico de diafragma }\end{array}$ & $\begin{array}{l}\text { Estudio de sensores piezoeléc- } \\
\text { tricos en aplicaciones de medi- } \\
\text { ción de fuerza }\end{array}$ & $\begin{array}{l}\text { Acxel Fernández } \\
\text { Galeano }\end{array}$ & $\begin{array}{l}\text { Carga de condensador } \\
100 \mathrm{nF} \\
\text { Voltaje máximo: } \\
1400 \mathrm{mV}\end{array}$ \\
\hline $\begin{array}{l}\text { Simulación de una ca- } \\
\text { minata de } 3,5 \text { millas/h } \\
\text { en una caminadora }\end{array}$ & $\begin{array}{l}\text { Diseño de un colector de ener- } \\
\text { gía piezoeléctrico mediante } \\
\text { optimización topológica que } \\
\text { maximice la transformación de } \\
\text { energía mecánica en eléctrica } \\
\text { generada por un ser humano } \\
\text { al caminar }\end{array}$ & $\begin{array}{l}\text { Esteban } \\
\text { Sepúlveda } \\
\text { Orozco }\end{array}$ & $\begin{array}{l}\text { Carga de batería NiMH } \\
20 \text { mAh } 1,2 \text { V en } 1 \text { hora y } \\
40 \text { minutos }\end{array}$ \\
\hline $\begin{array}{l}\text { Simulación de una ruti- } \\
\text { na diaria de ejercicio en } \\
\text { una bicicleta elíptica }\end{array}$ & $\begin{array}{l}\text { Prototipo generador de energía } \\
\text { eléctrica por el uso de una bi- } \\
\text { cicleta elíptica para la ilumina- } \\
\text { ción de un ambiente de deporte }\end{array}$ & $\begin{array}{l}\text { Kenny Palomino } \\
\text { Vera }\end{array}$ & $\begin{array}{l}\text { Carga de batería de } \\
4000 \text { mAh a } 6 \text { V en } \\
8 \text { horas y } 30 \text { minutos }\end{array}$ \\
\hline
\end{tabular}

Fuente: Ahmad y Hashim (2011), Fernández Galeano (2018), Sepúlveda Orozco (2014) y Palomino Vera (2016)

Elaboración propia 


\section{CONCLUSIONES}

- La elaboración de un sistema de captación de la energía que se da al caminar es técnicamente viable, mediante el uso del fenómeno de la piezoelectricidad como fuente de energía limpia, para la captura de la energía que se pierde al caminar. Se escogió el calzado como aplicación, teniendo en cuenta que una de las principales actividades del ser humano es caminar.

- En la etapa de sistema de captación de energía se utilizaron los sensores piezoeléctricos PZT de diafragma cerámico tipo tweeter que está diseñado para generar sonido y no son ideales para generación de energía.

- Al pisar, el sistema de once sensores piezoeléctricos genera energía que no está lista para ser usada por su carácter de corriente alterna. Por esta razón, en la etapa de acondicionamiento se rectificó y amplificó la corriente alterna generada, como un puente de diodos, y con un condensador se mantuvo el voltaje estable para no dañar la unidad de almacenamiento con las posibles variaciones de tensión.

- En la etapa de almacenamiento, la unidad que se utilizará para el almacenamiento de la energía puede variar según la aplicación y la capacidad de esta. Las unidades de almacenamiento más utilizadas son los condensadores, supercondensadores y baterías.

- El prototipo de la plantilla fue elaborado en tres fases para trabajar con las medidas reales del pie de una persona. Se empleó la técnica de digitalización de escaneo 3D con el HandySCan 700 3D y el software VXscan para recopilar la información dimensional y geométrica del pie de una persona talla 38, con el propósito de recrear un modelo tridimensional. La materialización de los prototipos se hizo con la tecnología de impresión 3D. Se utilizó la impresora Replicator $2 x$ de la marca Makerbot y el filamento flexible PLA de la misma marca. El resultado fue una estructura rígida que no presenta la característica de maleabilidad que tienen las plantillas comunes. Además, no permitió la ubicación de los sensores piezoeléctricos en los espacios circulares que se diseñaron.

- La prueba del encendido del diodo LED se hizo para comprobar que el circuito propuesto genera energía. Al simular la caminata de una persona, se logró encender el diodo y se generó, aproximadamente, hasta $1 \mathrm{~V}$ con cada pisada.

- No se generó suficiente energía para cargar una unidad de almacenamiento como se esperaba. Generalmente, para cargar la batería de un celular se necesitan, aproximadamente, $3000 \mathrm{mAh}$ y $5 \mathrm{~V}$. Ni para la carga de condensador de menor capacidad que requiere de $10 \mu \mathrm{F} \mathrm{y} 6 \mathrm{~V}$. 


\section{RECOMENDACIONES}

- Como parte de la etapa de la elección del sistema de captación de energía, se puede usar como ventaja la combinación y complementación de diversas fuentes de captación de energía para una mayor generación de energía limpia. También, en caso de contar con un buen presupuesto, se podrían emplear sensores de naturaleza piezoeléctrica y componentes electrónicos profesionales de alta calidad.

- Antes de empezar con la investigación, se desconocían los materiales y sustancias químicas que se utilizaron para la fabricación de los sensores piezoeléctricos. Es importante que, para posteriores investigaciones, se utilicen sensores artificiales sin plomo o sin otras sustancias dañinas para el medio ambiente y las personas involucrados en su fabricación.

- Revisar las hojas de especificaciones de los sensores piezoeléctricos antes de trabajar con ellos, al igual que de los componentes electrónicos.

\section{REFERENCIAS}

Ahmad, R., y Hashim, M. H. (2011). Development of energy harvesting device using piezoelectric material. 2011 Fourth International Conference on Modeling, Simulation and Applied Optimization, 1-6. https://doi.org/10.1109/ICMSA0.2011.5775592

Dewangan, S. K., y Dubey, A. (2017). Design and implementation of energy harvesting system using piezoelectric sensors. International Conference on Intelligent Computing and Control Systems, ICICCS 2017, 598-601. https://doi.org/10.1109/ ICCONS.2017.8250532

Fan, K., Liu, Z., Liu, H., Wang, L., Zhu, Y., y Yu, B. (2017). Scavenging energy from human walking through a shoe-mounted piezoelectric harvester. Applied Physics Letters, 110(14). https://doi.org/10.1063/1.4979832

Fernández Galeano, A. E. (2018). Estudio de sensores piezoeléctricos en aplicaciones de medición de fuerza (tesis de grado). Universidad Politécnica de Valencia, Escuela Técnica Superior de Ingeniería del Diseño, Valencia, España. Recuperada de https://riunet.upv.es/bitstream/handle/10251/111211/Fern\%C3\%A1ndez\%20 -\%20Estudio $\% 20$ de $\% 20$ sensores $\% 20$ piezoel\%C3\%A9ctricos $\% 20$ en $\% 20$ aplicaciones $\% 20$ de $\% 20$ medici $\%$ C $3 \%$ B 3n $\% 20$ de $\% 20$ fuerza.pdf? sequence $=3 \&$ isAllowed $=y$

Fluke Corporation. (2020). ¿Qué es la capacitancia? Recuperado de https://www. fluke.com/es-pe/informacion/mejores-practicas/aspectos-basicos-de-lasmediciones/electricidad/que-es-la-capacitancia 
Jiménez Martínez, F. J., de Frutos, J., Alonso, D., y Vázquez, M. (2015). Optimización de piezoeléctricos comerciales para su uso en sistemas de energy harvesting. Boletín de la Sociedad Española de Cerámica y Vidrio, 54(6), 247-253. https://doi. org/10.1016/j.bsecv.2015.09.001

Maiwa, H. (2016). Piezoelectric energy harvesting. En T. Ogawa (Ed.), Piezoelectric Materials. https://doi.org/10.5772/64162

Mezei, F. (2011). Basics concepts. En T. Imae, T. Kanaya, M. Furusaka y N. Torikai (Eds.), Neutrons in soft matter. https://doi.org/10.1002/9780470933886.ch1

Palomino Vera, K. A. (2016). Prototipo generador de energía eléctrica por el uso de una bicicleta elíptica para la iluminación de un ambiente de deporte (tesis de grado). Universidad Tecnológica del Perú. Recuperada de https://hdl.handle.net/20.500.12867/237

Sepúlveda Orozco, E. (2014). Diseño de un colector de energía piezoeléctrico (energy harvesting) mediante optimización topológica que maximice la transformación de energía mecánica en eléctrica generada por un ser humano al caminar (tesis de maestría). Universidad Nacional de Colombia, Colombia. Recuperada de https:// repositorio.unal.edu.co/handle/unal/52878

Twenergy. (2019). Almacenamiento de energía. ¿Qué son los supercondensadores y cuáles son sus aplicaciones? Recuperado de https://twenergy.com/ eficiencia-energetica/almacenamiento-de-energia/supercondensadores-queson-aplicaciones/

Vishay Semiconductors. (2020). High Intensity LED, Ø 5 mm Clear Package. Recuperado de https://www.vishay.com/docs/83004/tldr5800.pdf

Xie, L., y Cai, M. (2015). An in-shoe harvester with motion magnification for scavenging energy from human foot strike. IEEE/ASME Transactions on Mechatronics, 20(6) https://doi.org/10.1109/TMECH.2015.2428618 